\title{
Vibrosight++: City-Scale Sensing Using Existing Retroreflective Signs and Markers
}

\author{
Yang Zhang \\ Carnegie Mellon University \\ Pittsburgh, PA, USA \\ yang.zhang@cs.cmu.edu
}

\author{
Sven Mayer \\ Carnegie Mellon University \\ Pittsburgh, PA, USA \\ info@sven-mayer.com
}

\author{
Jesse T. Gonzalez \\ Carnegie Mellon University \\ Pittsburgh, PA, USA \\ jtgonzal@cs.cmu.edu
}

\author{
Chris Harrison \\ Carnegie Mellon University \\ Pittsburgh, PA, USA \\ chris.harrison@cs.cmu.edu
}

\begin{abstract}
Today's smart cities use thousands of physical sensors distributed across the urban landscape to support decision making in areas such as infrastructure monitoring, public health, and resource management. These weather-hardened devices require power and connectivity, and often cost thousands just to install, let alone maintain. In this paper, we show how long-range laser vibrometry can be used for low-cost, city-scale sensing. Although typically limited to just a few meters of sensing range, the use of retroreflective markers can boost this to $1 \mathrm{~km}$ or more. Fortuitously, cities already make extensive use of retroreflective materials for street signs, construction barriers, road studs, license plates, and many other markings. We describe how our prototype system can co-opt these existing markers at very long ranges and use them as unpowered accelerometers for use in a wide variety of sensing applications.
\end{abstract}

\section{CCS CONCEPTS}

- Human-centered computing $\rightarrow$ Ubiquitous and mobile computing; Ubiquitous and mobile computing systems and tools;

\section{KEYWORDS}

Laser Vibrometry, Smart City, Internet-of-Things, Ubiquitous Computing, Sensing

\section{ACM Reference Format:}

Yang Zhang, Sven Mayer, Jesse T. Gonzalez, and Chris Harrison. 2021. Vibrosight++: City-Scale Sensing Using Existing Retroreflective Signs and Markers. In CHI Conference on Human Factors in Computing Systems (CHI '21), May 8-13, 2021, Yokohama, Japan. ACM, New York, NY, USA, 14 pages. https://doi.org/10.1145/3411764.3445054

\section{INTRODUCTION}

Urban environments are vast and complex, with a wide range of facets that city planners, public transit agencies, business owners, citizens, and many other stakeholders might wish to sense [17, 22, $41,54]$. This sensor data can be used by cities to increase mobility [5], improve operational efficiency [38], boost quality of life [41], and become more sustainable [29]. For this reason, the concept of smart cities is gaining traction, but due to high cost, city-scale

\section{(c) (i) $\odot$}

This work is licensed under a Creative Commons

Attribution-NonCommercial-NoDerivs International 4.0 License.

CHI '21, May 8-13, 2021, Yokohama, Japan

(c) 2021 Copyright held by the owner/author(s).

ACM ISBN 978-1-4503-8096-6/21/05.

https://doi.org/10.1145/3411764.3445054 deployments are rare today except in mature and narrow use cases, such as traffic flow [53, 55] and gunshot detection [8, 46]. This leaves an exceptionally long tail of small, but interesting smart city applications with no viable source of data and without budgets to put them into practice.

The primary reason for such high costs is that today's smart city sensing paradigm is built around massive deployment of physical sensing infrastructure. One notable example effort is Chicago's Array of Things project [2], launched in 2015. Backed by at least $\$ 4 \mathrm{M}$ in funding, the project has deployed 130 sensing nodes on poles through the city as of January of 2020. In general, these smart city nodes are weather-hardened devices requiring power and connectivity, and cost thousands to install on a pole, not including hardware and maintenance costs, which is almost certainly many times more expensive. Thus, the central thesis of our research is that to unlock the potential of smart cities, a truly tectonic shift in city-scale sensing is needed to 1) dramatically reduce deployment costs, 2) virtually eliminate maintenance, and 3) generalize across a wide array of sensing applications that can improve cities. Today, 4.1 billion people - a majority of the world's population, live in urban areas, and thus cities occupy a central role in modern human life. Understanding cities is to understand ourselves, and if cities can be made smarter through sensors and computing, we can improve the quality of life for many people.

In this paper, we present one candidate method for ultra-widearea sensing that could be applied to the expansive urban landscape and meet our aforementioned three criteria. More specifically, we suggest a novel application of laser vibrometry, used extensively in fields such as industrial quality control, aeronautics, and structural health monitoring [14, 30, 35]. These sensors (described in greater detail later) are generally used to sense at a few meters range. However, the sensing range can be significantly increased by attaching a retroreflective sticker (or equivalent marker) to a surface of interest, which greatly increases the returned laser light and permits accurate measurement. Importantly, the "smarts" (and expense) is limited to the vibrometer itself (e.g., the entry-level Polytec VibroGo costs around \$30k [42]), with retroreflective tape costing less than 1 USD per yard. Of course, given the number of facets we might wish to sense across a city, this approach does not necessarily meet our first criteria (i.e., having to go out and specially tag all of these objects would drive up deployment cost).

The second key insight that drove this research project was the fact that many cities, very fortuitously, have already deployed retroreflectors by the tens of thousands. Street signs, construction barriers, road studs, lane stripes, traffic control signage, license plates, bicycle retroreflectors, and many other objects in the urban domain are made with retroreflective materials to improve visibility 


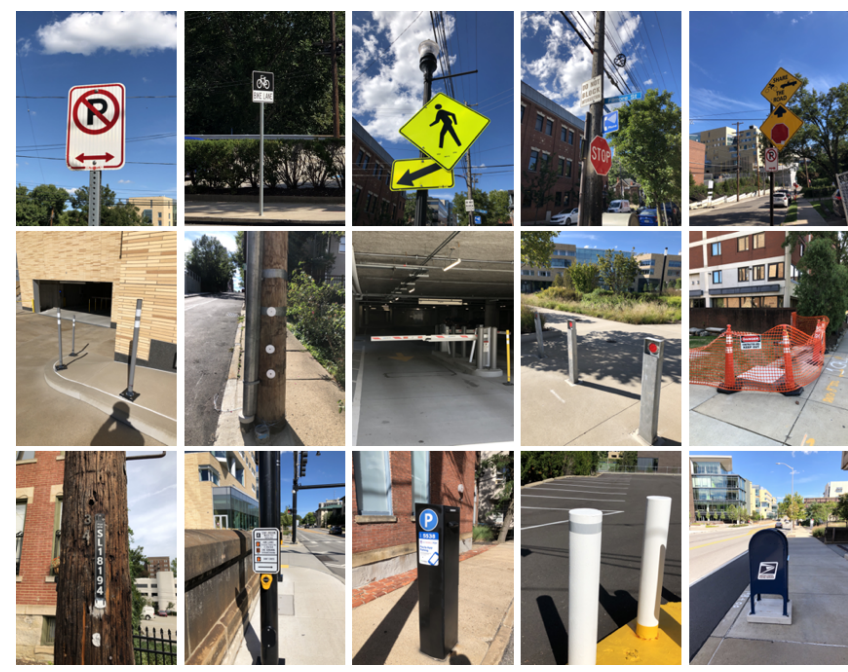

Figure 1: Examples of retroreflective signs and markers that exist in urban environments.

at night. Figure 1 offers a small sampling of the items we found during our explorations. In a five-city survey we conducted, described later, we found an average density of $\sim 7000$ retroreflectors per square kilometer. In essence, cities have already unknowingly invested and built out all of the sensing infrastructure our approach needs.

Our approach allows these existing retroreflectors (requiring no power or connectivity) to be functionally appropriated as lowresolution accelerometers at extreme ranges. As much prior work has shown, accelerometers can be rich signal sources used to power a wide range of domestic and commercial activity recognition tasks, e.g., $[27,28]$. We show how many of the same techniques can be applied to the civic domain, and we showcase eleven example uses to illustrate the potential and generalizability of our approach, meeting our third criteria. Before that, we describe the core concepts that underpin the physics of our approach and review key related work. We then describe our proof-of-concept system, which cost us around $\$ 600$ to build (much less in volume production), before moving to an evaluation that validates our sensing approach. Finally, we conclude this work with a wide variety of smart city applications enabled by our sensing technique.

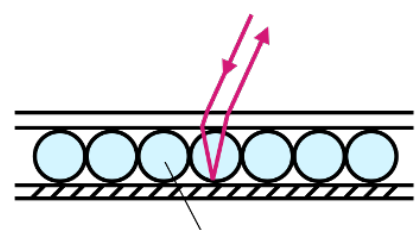

Glass beads

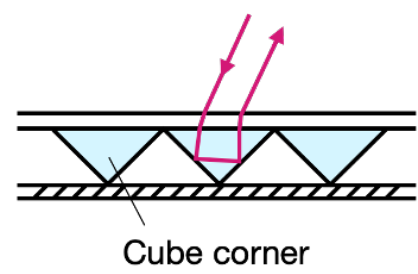

Figure 2: Two typical micro-structures of retroreflective materials: beads and corner reflectors.

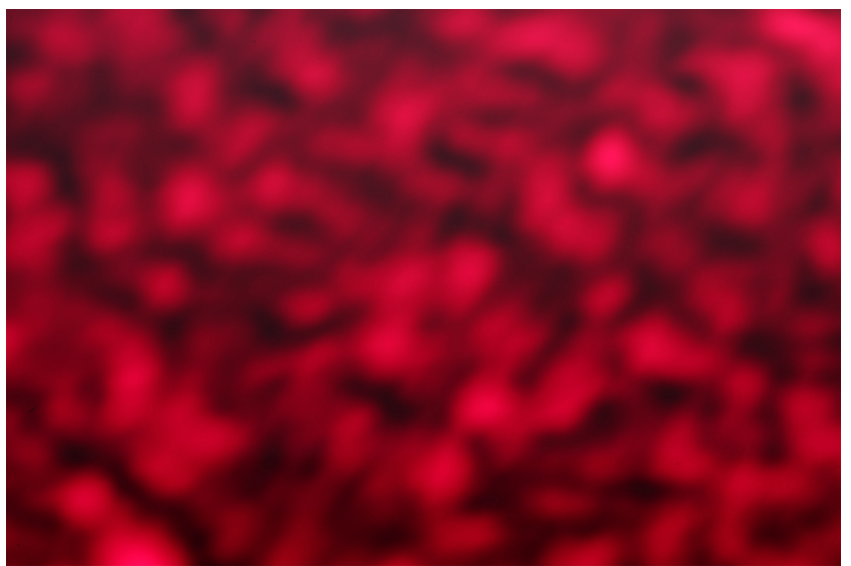

Figure 3: Speckle pattern reflected off of a remote retroreflective marker as captured by an exposed CCD (i.e., no focus optics).

\section{PRINCIPLES OF OPERATION}

As a primer, we quickly review some relevant physics. Traditional reflectors, like a mirror one might have at home, bounce energy at the same angle at which a ray approaches the surface, mirrored across the surface normal. In contrast, retroreflectors return energy back along the exact same vector as the source. This ability to return energy towards the source of emission is particularly useful for urban signage and markers, where illumination from vehicle headlights makes them highly visible without the need for powered artificial lighting at the sign (i.e., signs can be totally passive). There are two general types of retroreflector geometry used for wavelengths in and around the visible light spectrum (Figure 2). The first are spherical beads, made of glass or plastic, which have a carefully formulated index of refraction such that light escapes the sphere at the same angle of incidence. A second bead variety uses half-mirrored spheres to achieve retroreflection. Finally, retroreflectors made from right-angle prisms (also called corner reflectors) are very common. For a more in-depth review on the physics of reflection, we recommend [7].

There are two main types of laser vibrometry. First are ones built around interferometry $[9,18]$, where minute surface vibrations of an object cause the laser light to return at a slightly different phase than the emitted signal, which is used as a reference. Mixing these two signals together (using sophisticated internal optics) creates constructive and destructive interference, which can be read by a sensitive photodetector. The second type of laser vibrometry takes advantage of the reflected laser speckle pattern [57] (Figure 3). Object vibrations cause the reflected pattern to oscillate, and thus a photosensor placed in the light field will observe the speckle pattern translating through its field of view, causing the received intensity to rise and fall. This AC signal encodes an object's vibrations, and the DC component is useful for detecting the presence or occlusion of retroreflectors in the environment. For our proof-of-concept hardware, we use the intensity-based approach, but both types of laser vibrometry are applicable. 
Table 1: Results from our retroreflective marker survey of two-block regions in five cities.

\begin{tabular}{|c|c|c|c|c|c|c|c|c|c|}
\hline City & On Structure & On Pole & On Vehicle & On Tree & On Road & On Misc. & $\begin{array}{l}\text { Total } \\
\text { Count }\end{array}$ & $\begin{array}{c}\text { Area } \\
\left(m^{2}\right)\end{array}$ & $\begin{array}{c}\text { Density } \\
\left(\text { count } / \mathbf{k m}^{2}\right)\end{array}$ \\
\hline Ann Arbor, USA & 0 & 104 & 89 & 3 & 0 & 0 & 196 & 45491.0 & 4308.5 \\
\hline Enfield, UK & 5 & 52 & 173 & 0 & 0 & 0 & 230 & 26990.5 & 8521.5 \\
\hline Harbin, China & 17 & 11 & 99 & 0 & 11 & 0 & 138 & 47430.1 & 2909.5 \\
\hline Pittsburgh, USA & 24 & 188 & 65 & 4 & 39 & 8 & 328 & 33038.3 & 9927.9 \\
\hline Stuttgart, Germany & 11 & 103 & 71 & 0 & 6 & 1 & 192 & 19863.2 & 9666.1 \\
\hline
\end{tabular}

\section{RETROREFLECTOR SURVEY}

While we anecdotally knew there were many retroreflective signs and markers deployed in cities, we wished to more formally catalog their uses and placements, and also derive an estimate of their density. For this, we surveyed two-block regions adjacent to the city centers of five mid-sized cities - one in Asia (i.e., Harbin), two in North America (i.e., Ann Arbor and Pittsburgh), and two in Europe (i.e., Enfield and Stuttgart). We only cataloged along public streets, and we did not survey any interior spaces, nor exterior spaces that existed inside of the street grid (e.g., surface parking lots). We also treated vehicles as a special category, as they often feature seven or more discrete retroreflectors (depending on local law, e.g., [13]). Rather than counting these as separate retroreflectors, we count them as one in our tally.

In total, we found 1084 retroreflectors in a cumulative area of $172,813 / \mathrm{m}^{2}$ (which includes block interiors which we did not survey). This equates to an average retroreflector density of $6272.7 / \mathrm{km}^{2}$. In our hometown, Pittsburgh, a mid-sized city of $\sim 150 \mathrm{~km}^{2}$, it means it is likely there is on the order of a million deployed retroreflectors, each of which is a potential smart city sensor.

Figure 1 shows representative retroreflectors we found during our survey. We categorized retroreflectors into six categories: 1) Retroreflectors placed on structures, such as buildings, covered bus stops, and phone booths. 2) Retroreflectors on poles, such as traffic light and power line poles, as well as smaller poles used for signs (e.g., bus stop, yield, parking information, one-way street). 3) Retroreflectors on vehicles, which are a safety requirement that allow cars to be seen even when their lights are off. 4) Retroreflectors on trees, which are used to reduce collisions, but seem comparatively rare. 5) Retroreflectors attached to roads, such as road studs and fixed bollards, most often to help demark traffic flow (we note that painted road markings are often retroreflective, but we did not include them in this tally). Finally, 6) we found some miscellaneous retroreflectors that did not fit neatly into our previous categories, but what might be broadly described as "street furniture" [50] (e.g., retroreflectors attached to parking payment kiosks, trash dumpsters, fire hydrants, and mailboxes). Table 1 provides a breakdown of these tabulations.

\section{RELATED WORK}

Our work mainly intersects with three prior research areas: physically instrumenting objects in the urban domain, wide-area sensing approaches, and long-distance sensing specifically using lasers.

\subsection{Instrumenting Urban Objects with Sensors}

Perhaps the most straightforward sensing approach is to directly instrument urban objects of interest. Vibrations and accelerations of infrastructure and other urban assets have already been shown to be useful for smart city applications $[15,40]$. Researchers have also instrumented buildings and bridges with piezoelectric-based sensors to monitor their structural health $[39,45]$. It is also possible to use piezoelectric sensors on roads for traffic flow monitoring [31], which has seen some commercial success [37]. Fiber Bragg grating sensors have also been embedded into infrastructure to sense vibrations for similar uses [36, 48]. Water flooding and ice sensors are also common, and have been installed on e.g., roadways and sewer lines to improve safety and intervention response time $[3,20,34]$.

\subsection{Wide-Area Sensing Approaches for Cities}

Since instrumenting objects with sensors can be costly to scale and maintain, it is common to leverage long-range sensors that can detect activities from afar and can cover wider areas. The most common approaches used today are microphones $[6,8,46]$ and cameras, increasingly powered by computer vision [26, 33, 44]. Researchers have also explored specialized sensors for air quality [43], water quality [10] and weather sensing $[19,56]$. Sensor fusion of multiple sensor types (e.g., airborne particulates, humidity, and temperature) has been shown to expand the range of detectable activities and events [21, 49, 52]. Although most sensors are deployed at static locations, researchers have also attached sensors to moving vehicles and drones, which significantly extends the coverage area [24, 51]. Satellites are also a powerful remote sensing tool that enables urban and wide-area applications such as weather forecasting, wildfire detection, terrain mapping, forest health sensing, and urban sprawl modeling $[11,16,32]$.

\subsection{Remote Sensing with Lasers}

More related to our sensing technique are previous systems which also employ lasers for remote sensing. Given their high coherence, lasers can achieve high signal fidelity at long distances. Practical uses of laser sensing include distance and angle measurement (e.g., surveying total stations), speed measurement (e.g., laser speed guns), and even depth sensing (e.g., LiDAR). Closer to our work are systems that leverage lasers to measure vibrations. Commercial laser vibrometers [42] use optical interferometry to capture vibrations of surfaces for e.g., structural examination and quality control purposes. In the research domain, prior work has explored laser vibrometry for indoor activity recognition $[47,57]$. This paper 
directly extends our own previous work on Vibrosight [57], which focused on indoor activity and appliance recognition at room-scale. The longest sensing range demonstrated by Vibrosight was less than $10 \mathrm{~m}$, whereas our new system works outdoors at hundreds of meters and hence the name of our system - Vibrosight++.

\section{VIBROSIGHT++ SYSTEM}

In this section, we describe our Vibrosight++ system, which includes hardware and software components working together to achieve our vision of long-range vibrometry sensing. Towards the end of our pipeline, we create several different processed signal streams, which are used to power different sensing applications.

\subsection{Hardware}

Our system (Figure 4) consists of two major hardware components: 1) a pan-tilt motor platform, and 2) a sensor bundle. The pan-tilt motor platform is set on a tripod and controls the angle of the sensor with a resolution of 0.00625 degrees - corresponding to a $10 \mathrm{~mm}$ step at a distance of 100 meters. This pan-tilt platform allows us to scan the environment for retroreflectors, and then cycle between them when multiple retroreflectors are present.

The sensor bundle consists of a $200 \mathrm{~mW} 650 \mathrm{~nm}$ laser, a $1000 \mathrm{~m}$ range finder, and a photodetector (Thorlabs PDA100A2). During the initial setup, both the photodetector and the range finder are calibrated to align with the laser (using small manual pan-tilt stages). We inserted an optical band-pass filter (Thorlabs FB650-40) in side a $13.5 \mathrm{~cm}$ matte black optical tube to make the photodetector more immune to unwanted ambient light (e.g., sunlight, reflections from windows, street lights, etc.). The output of the photodetector is amplified and sampled by a digital audio preamp (U-PHORIA UMC404HD) before being streamed to a laptop over USB for processing. The output of the range finder is also streamed to the laptop over USB.

In total, our sensing apparatus cost $\sim \$ 600$ to build as a one-off prototype. No doubt in commercial production it could be made many times less expensive and with superior SNR. A good vantage point with line-of-sight to many retroreflectors would likely equate to just a few dollars per sensed point.

\subsection{Eye Safety}

Our laser output power is $200 \mathrm{~mW}$ (Class III B), which is hazardous for eye exposure at short distances, but eye safe at distances longer than the Nominal Ocular Hazard Distance (NOHD) [23]. At constant laser power, NOHD is inversely proportional to laser divergence. As a safety measure, we adjusted the divergence of our laser in accordance with ANSI Z136.1 [1]. At our tightest divergence (1.62 milliradians), the NOHD is $95 \mathrm{~m}$. By defocusing our laser, we can make it eye safe down to around $46 \mathrm{~cm}$, where the energy density is under the recommended $2.5 \mathrm{~mW} / \mathrm{cm}^{2}$ exposure. There are two additional ways to improve safety. First, we envision strategic placement of our system at higher vantage points to remove the possibility of intersecting with traffic and pedestrians inside the NOHD. Finally, our system measures the reflected light intensity within 50 microseconds of laser power. This is well under the 250-millisecond exposure hazard threshold detailed in [1] if we are operating under $2.5 \mathrm{~mW} / \mathrm{cm}^{2}$. We use this 50 -microsecond interval

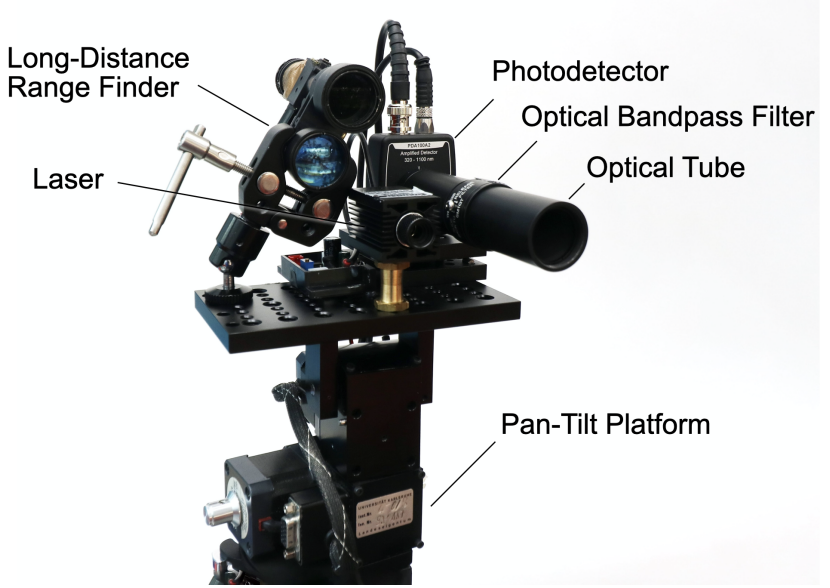

Figure 4: Our system consists of several hardware components: a laser, range finder, photodetector, optical tube with bandpass filter, and a pan-tilt motor platform.

in our retroreflector search (described in the next section), meaning even accidental contact with humans is non-damaging. Likewise, when we are capturing signal from known retroreflectors, we can easily detect if they are occluded (e.g., by a car or human), and we turn off the laser (again within 50 microseconds) and cycle to the next target.

\subsection{Environmental Conditions}

Environmental conditions such as illumination and weather (e.g., snowing, raining) can affect our signal. Although we can deliberately leverage these signals for weather sensing (as we will show later in the paper), we generally consider these effects to be noise. Our optical design includes a bandpass filter (centered on our laser light wavelength) and the optical tube mitigates indirect rays (e.g., sun, street lights). Even still, our system shows higher SNR (i.e., $\sim 1.6 \mathrm{~dB}$ improvement) at night when there is nearly no ambient light than when our system is operating under direct sunlight. Cloudy weather results in SNRs somewhere between these two extremes. In the future, measured ambient illumination level could be used to calibrate detection pipelines. Weather conditions like snowing and raining result in transient occlusions of the laser beam. For vibration and motion sensing, this interference cannot be easily mitigated, and requires additional research. Until this issue is overcome, sensing can be suspended until weather clears to avoid false positives.

\subsection{Retroreflector Search}

In order to leverage existing signs and markers for sensing, we must first find them. After our system is deployed in an environment, we define a region of interest by specifying azimuth and elevation ranges. Our system then raster scans this region row-by-row, with an angular resolution of 0.05 degrees. At each angle, we measure distance with the rangefinder in order to generate a depth map (Figure 5, top). After the distance measurement, we measure the ambient light level using our photodetector. We then turn on the 

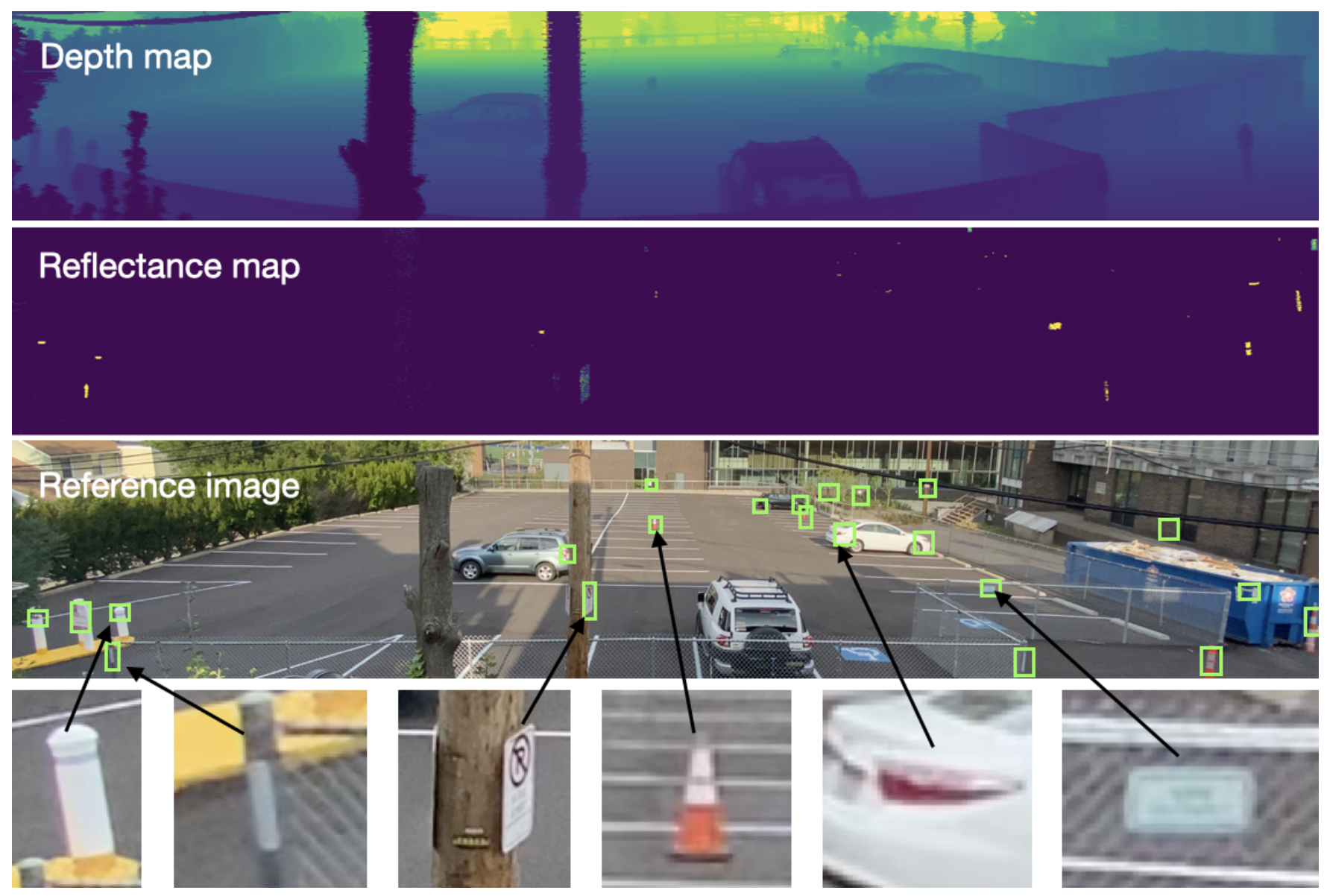

Figure 5: Depth map, reflectance map, and reference image of a parking lot where we conducted the retroreflector search study. Green rectangles denote found retroflectors.

laser for 50 microseconds and take a second reading. We use the difference between these two values to create a reflectance map (Figure 5, second from top).

Our retroreflector search algorithm uses both the depth map and the reflectance map. We begin with a noise reduction process by using the depth map to calibrate the raw reflectance values (i.e., points further away are scaled up to compensate for light intensity loss over distance). We then eliminate all reflectance values under a predetermined threshold, and run a mean-shift clustering algorithm on the remaining points.

Figure 5 offers an example of our retroreflector search. In this example, we deployed our sensor on the second story of a commercial building, pointing at a parking lot area roughly 1800 square meters in size. We defined a scan scope of 110 azimuth degrees by 16 elevation degrees, and started the retroreflector search. After the search completed, we compared our results with our manually logged ground-truth counts and locations of the retroreflectors in the environment. Our system correctly found 22 out of 29 retroreflectors in the environment. Two of the missed items were oriented at extreme angles relative to our rig; while they did register on the reflectance map, the sensed light was not bright enough to avoid being removed by thresholding. The other five items were found, but due to tight spacing, were clustered together into two items as opposed to five separate retroreflectors, which we counted as errors.

\subsection{Presence/Occlusion Sensing}

To sense the presence and occlusion of retroreflectors, we used a Teensy 4.1 (built-in ADC) to measure the output voltage of the photodetector. A resistor is used to downscale the photodetector output to the dynamic range of the Teensy ADC. Each measurement takes 50 microseconds to complete. These measurements are then thresholded for presence/occlusion detection, and we use a small voting window to add stability to the output. We found this straightforward threshold-based detection to work well in practice.

\subsection{Vibration and Motion Sensing}

Vibration and motion sensing requires a higher sampling rate. For this, we used an USB audio preamp (U-PHORIA UMC404HD) that samples the photodetector output at $44.1 \mathrm{kHz}$. This output is bandpass filtered at different frequency bands for different applications. The filtered output contains vibration and movement information 

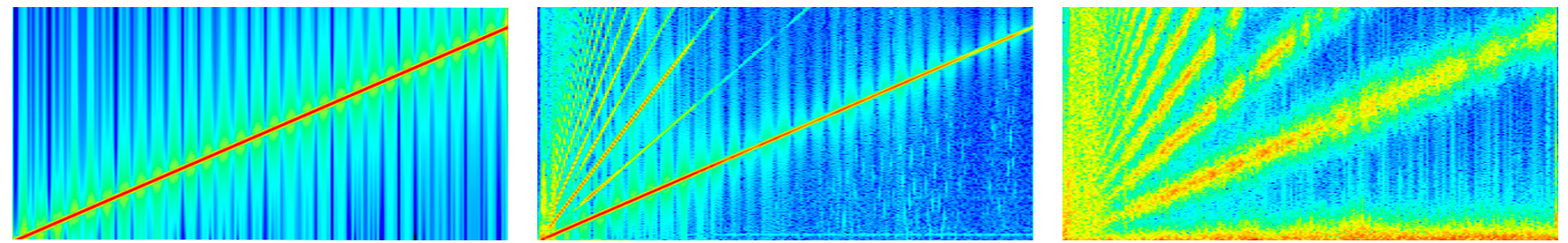

Figure 6: Spectrogram of a $2 \mathrm{~Hz}-2 \mathrm{kHz}$ swept-frequency signal (left), recorded signal from an accelerometer affixed to the speaker diaphragm (center), and from Vibrosight++ at $128 \mathrm{~m}$ pointing at a retroreflective marker on the speaker diaphragm (right). All spectrograms in this paper are in log scale.
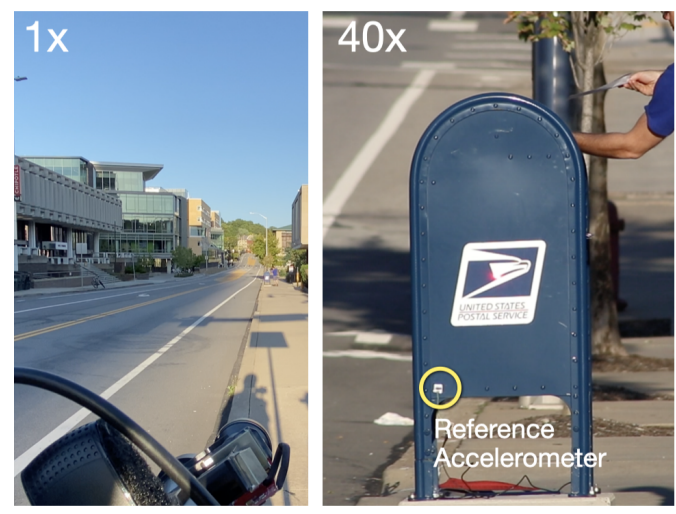

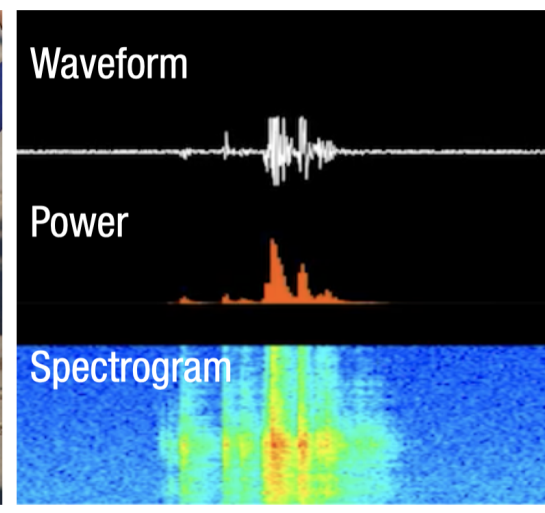

Ref. Accelerometer

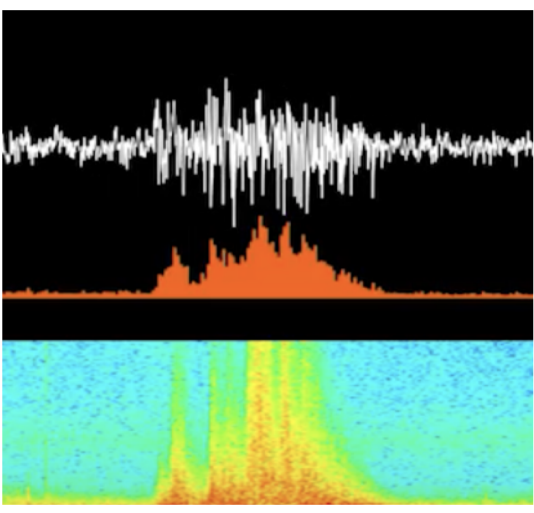

Vibrosight++

Figure 7: Sensing the vibrations of a mailbox when someone deposits a letter with a powered accelerometer attached to the mailbox and with Vibrosight++ 60m away.

that can be directly used for applications that rely on time-domain signals. For example, it is possible to simply count the peaks of the vibration signal induced on road paint for traffic monitoring. For applications that require differentiation of events using frequencydomain characteristics (e.g., different vehicles with different engines), we compute an FFT (window size $=2048$ ) and also maintain a rolling spectrogram. We also sum the non-DC components as an indicator of total vibrational power, which can be used to trigger thresholds in some monitoring applications. For our own applications that required more complex differentiation of signals, we used standard machine learning techniques (i.e., SVM with linear kernel) for classification and regression.

\subsection{Comparison to Accelerometer}

To investigate our system's accuracy in sensing vibration and motion, we conducted a comparison study with a gold standard sensor: a physical, powered accelerometer. To run this experiment, we used a large diaphragm speaker $(54 \mathrm{~cm}$ diameter) powered by a $600 \mathrm{~W}$ amplifier to output a swept frequency from $2 \mathrm{~Hz}$ to $2 \mathrm{kHz}$. We physically attached an accelerometer (Analog Devices ADXL335) and retroreflective tape (3M 79961 Scotchlite) to the center of the diaphragm. The analog accelerometer was read by the USB audio preamp at $44.1 \mathrm{kHz}$, while our laser vibrometry setup measured vibration using the retroreflective tape at a distance of $128 \mathrm{~m}$. Figure 6 illustrates the result; the main sweep is clearly visible and linear. Note the harmonics seen in both the accelerometer and Vibrosight++ signals are due to the physical design of the speaker, which cannot output a pure sine wave without secondary oscillations.

As a complement to our controlled experiment, we also selected one real-world example. We affixed an accelerometer to a mailbox and recorded the signal in the same manner as above. The USPS logo was already retroreflective, and so we use that in conjunction with our system at $60 \mathrm{~m}$. The signal of someone depositing a letter into the mailbox is shown in Figure 7 (see also Video Figure).

In both experiments, we found that although the Vibrosight++ signal is considerably more coarse and noisy than an actual accelerometer, the fidelity is more than sufficient to power most uses (e.g., it is very apparent when the mailbox door is opened). Additionally, we found that our vibrometer signal has persistent lowfrequency components due to ambient vibrations and optical noise, which we remove with digital bandpass filters in our later applications. Overall, we believe this to be a very strong result given that our system is sensing at a distance (vs. being affixed to the object and requiring local power).

\section{EVALUATION}

There are many factors that affect the sensitivity of our system, including retroreflector type, laser color, retroreflector color, retroreflector angle, and distance from the sensor. It was not possible to 

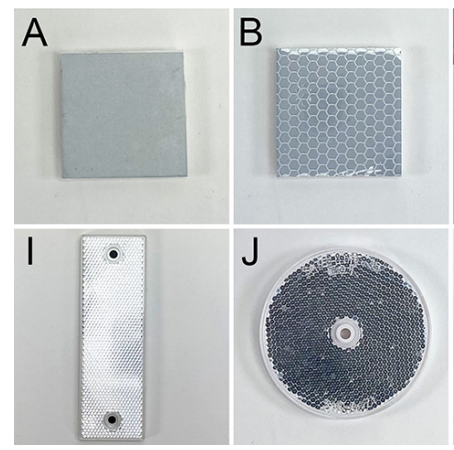
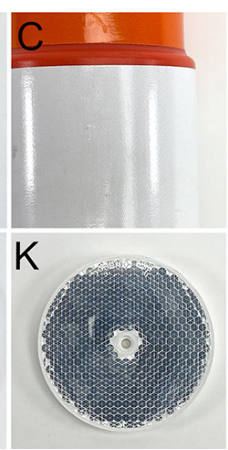
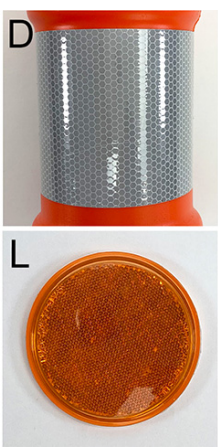

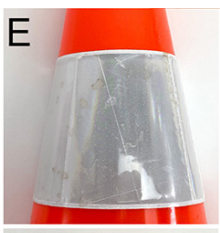

M

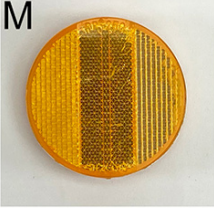

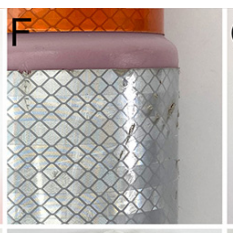
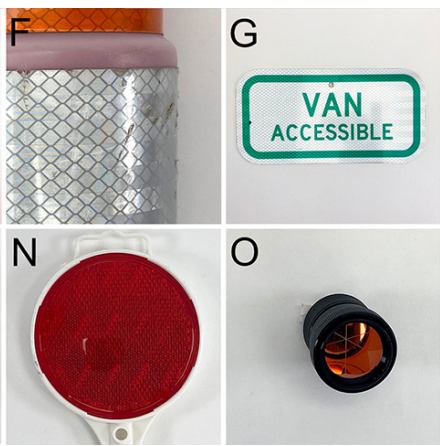

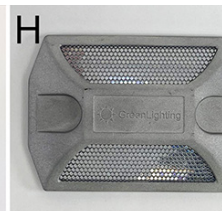

O

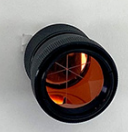

$\mathrm{P}$

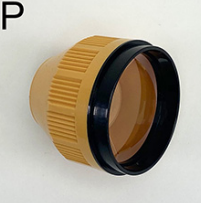

Figure 8: The 16 exemplary retroreflectors we used in our evaluations - $A$ to $G$ are micro-prism tapes and films, $H$ to $\mathrm{N}$ are retroreflective prism arrays, and $O$ and $P$ are large single prisms. We selected $A, B, E$, and $M$ for our in-depth motion and vibration evaluation.

test all combinations of these parameters, and so instead, we investigated each factor sequentially. Our data was collected in daylight, between midday and late afternoon. While great care and effort was spent to ensure all measurements were as comparable as possible, operating in an uncontrolled outdoor environment meant some variability in our measurements was unavoidable. Small inconsistencies arose from e.g., the sun changing angle over the duration of an experiment, variations in haze, and even light paths moving or oscillating due to air turbulence and temperature variation (i.e., mirages). Measured ambient illumination varied from 52 to 84 klux over the course of our experiments. Nonetheless, the main trends are readily apparent (e.g., signal decreases as retroreflector angle increases), and thus we encourage readers not to place emphasis on any one reading.

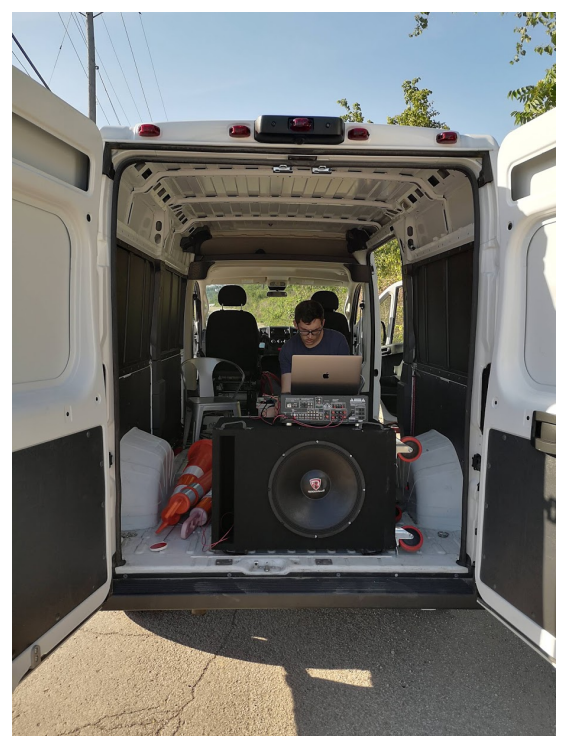

Figure 9: We loaded our signal generation apparatus into a van for mobility, which allowed us to test various distances in outdoor conditions.

\subsection{Test Retroreflectors}

We selected 16 markers and retroreflective materials (Figure 8) that were representative of the wide variety of retroreflective we found in our survey. Retroreflector A and B are commercial retroreflective tapes that can be adhered to any surface. $\mathrm{C}$ through $\mathrm{F}$ are commercial traffic cones and bollards that have a tape-like reflective material bonded to their surface. $G$ is an aluminum sign with a retroreflective layer (also tape-like). $\mathrm{H}$ is a metal-bodied road stud with an inset plastic retroreflector of small prisms. I through $\mathrm{N}$ are also molded plastic prism arrays (various sizes, shapes, colors, and quality). Finally, $\mathrm{O}$ and $\mathrm{P}$ are large prism retroreflectors typically used in surveying. As described in Principles of Operation, there are also two common ways to make retroreflective materials, using beads or prisms. For reference, A-E uses beads, while retroreflectors F-P use prisms. In general, prism-based retroreflectors are generally considered to be more efficient, which was confirmed with our later tests. We also found that signals from the same type of retroreflectors varied quite a lot across construction quality (plastics used, mirror surface finish, etc.).

\subsection{Test Apparatus}

To provide a controlled motion and vibration signal, we use the same $54 \mathrm{~cm}$ diameter speaker, powered by a $600 \mathrm{~W}$ amplifier, as described in Section 5.7. As before, different retroreflectors were affixed to the center of the speaker, and actuated with signal driven by a laptop. Given that our tests ranged up to 920 meters, in outdoor conditions, we decided to load our signal generation apparatus into a van for mobility (Figure 9). The engine was turned off during tests so as not to introduce any extraneous vibrations.

\subsection{Laser Color}

To explore if wavelength of light had a significant effect on SNR, we tested three different $200 \mathrm{~mW}$ lasers: red $(650 \mathrm{~nm})$, green $(532 \mathrm{~nm})$, and infrared $(940 \mathrm{~nm})$. Unlike the other experiments in this section, all measurements were completed without an optical bandpass filter. Retroreflector J (clear plastic) was used at a distance of $32 \mathrm{~m}$. We did not observe any significant difference in signal strength, measuring $0.15 \mathrm{~V}, 0.19 \mathrm{~V}$, and $0.17 \mathrm{~V}$ for the red, green and infrared lasers respectively. For all subsequent experiments, the red laser 


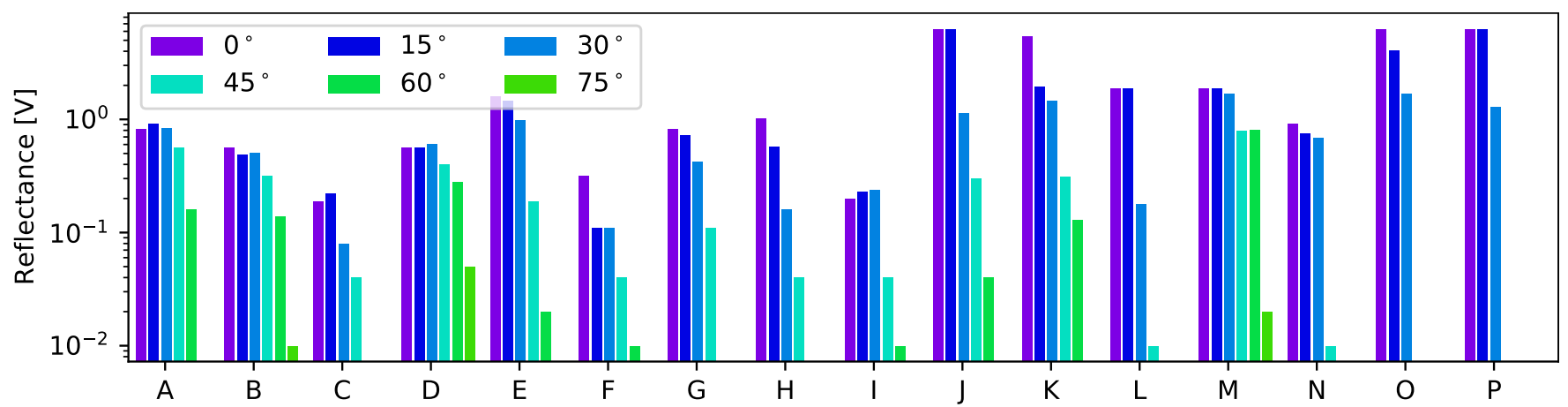

Figure 10: Reflection intensity of our 16 exemplary retroreflectors at 6 angles. Refer to Figure 8 for letter mappings.

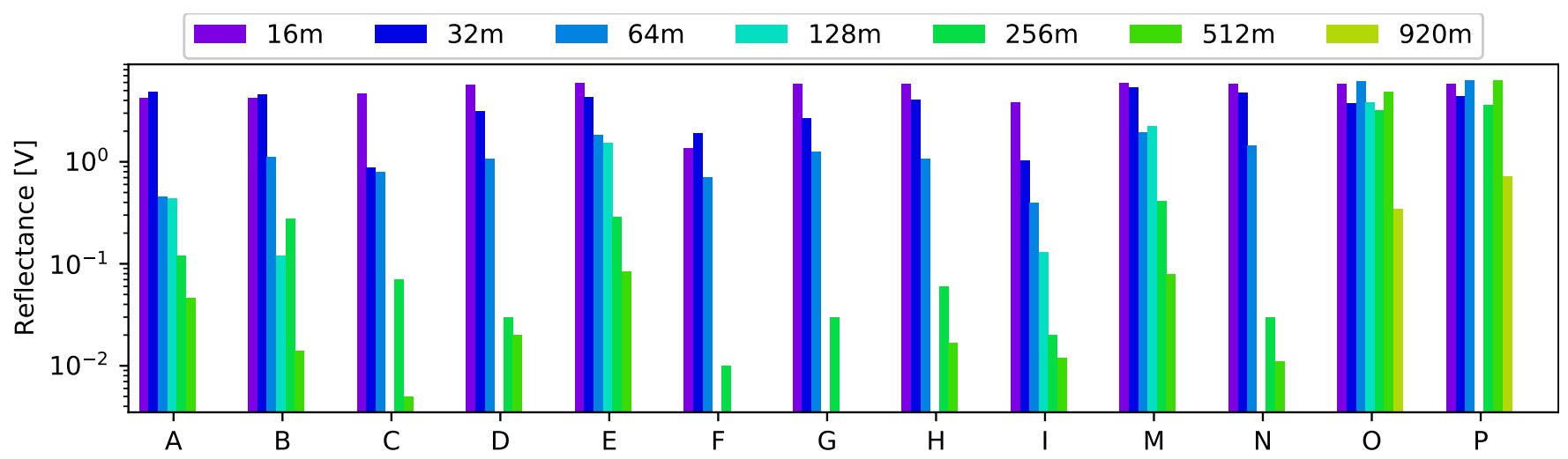

Figure 11: Reflection intensity of 13 retroreflectors at 7 distances. Refer to Figure 8 for letter mappings.

was used for ease of visual debugging. We note it may be ideal to use infrared lasers in the future, as they are invisible to humans.

\subsection{Reflector Color}

Although markers can be purchased in many different colors, they are most often red, orange, or clear (white) in urban use cases. This color effect is achieved with a semi-translucent, tinted plastic cover material, which passes a desired color through (and reflects it back out) but attenuates other wavelengths. For this test, we purchased a matched set of four colored retroreflectors. Using our red laser, we measured their reflectance at $32 \mathrm{~m}$. Unsurprisingly (given we are using a red laser), the red retroreflector had the strongest signal at $0.34 \mathrm{~V}$, followed closely behind by the clear $(0.24 \mathrm{~V})$ and orange $(0.23 \mathrm{~V})$ retroreflectors. Signal then drops off significantly as we move along the color spectrum, with the green retroreflector at $0.01 \mathrm{~V}$ and no measured signal with our blue retroreflector.

\subsection{Reflector Angle}

In general, the design of retroreflectors means that they are most efficient at reflecting when perpendicular to a light source, with reflectively dropping off as the angle of incidence increases. To measure this across our set of 16 exemplary retroreflectors, we investigated all angles between $0^{\circ}$ and $75^{\circ}$ in $15^{\circ}$ steps, where $0^{\circ}$ represent the laser being perpendicular to the retroreflector. We performed all angle tests at $32 \mathrm{~m}$ distance and indoors with consistent lighting conditions. As expected, we found the higher angle of incidence, the weaker the signal $\left(0^{\circ}\right.$ yields the highest reflectance and $75^{\circ}$ and beyond result in almost no reflectance; Figure 10).

\subsection{Distance - Presence/Occlusion}

For presence and occlusion detection, we wished to investigate the maximum distance at which we can detect the reflective signal (i.e., before it is subsumed into the noise floor). Not only does this test measure the quality of the retroreflectors, but also how well our sensor can pick up small changes in signal. Therefore, we had to leave our lab and find a long and flat private road where we could test the reflectance at different distances. The longest we could access was $920 \mathrm{~m}$ long. We tested reflectivity starting at $16 \mathrm{~m}$ and increasing by powers of two up to $512 \mathrm{~m}$. Instead of $1024 \mathrm{~m}$, we used $920 \mathrm{~m}$ as our farthest range. At each distance, we measured the photodetector output when the retroreflector was visible and occluded, and computed the difference (i.e., the magnitude of the reflected light). As expected, the reflection intensity decreases with distance (Figure 11). While all retroreflectors had detectable signals at distances up to $512 \mathrm{~m}$, only $\mathrm{O}$ and $\mathrm{P}$ yielded measurable reflections at $920 \mathrm{~m}$. Indeed, there is sufficient SNR to suggest that ranges of several kilometers should be possible with this retroreflector type. Finally, we note that recordings from three retroreflectors (i.e., J, 


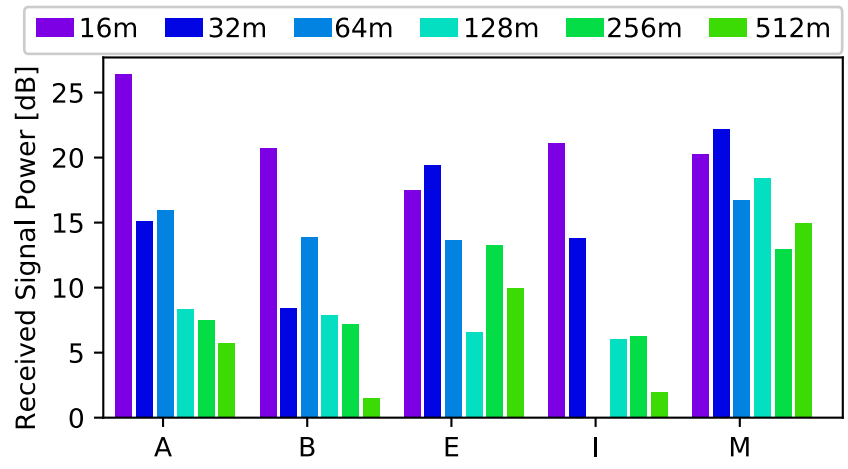

Figure 12: Received signal power from our distance-motion experiment using five of our exemplary retroreflectors. Refer to Figure 8 for letter mappings.

$\mathrm{K}, \mathrm{L}$ ) and from some other retroreflectors at the $128 \mathrm{~m}$ range were errorful and dropped from the graph, but the trend is still apparent.

\subsection{Distance - Motion}

We also investigated our system's sensitivity to motion at different distances. For this, we generated a sinusoidal displacement using our large speaker diaphragm $(0$ to $2 \mathrm{~Hz}$, in $0.5 \mathrm{~Hz}$ steps, three-second duration per step, with an average physical displacement of $4 \mathrm{~cm}$ ). We then cross-correlate the driver signal with the signal captured by Vibrosight++ to calculate the received signal power, the result of which is shown in Figure 12 for five representative retroreflectors. We found that our system is capable of detecting the motion signal from the retroreflectors at all distances up to $512 \mathrm{~m}$. Again, we found that received signal power attenuates as distance increases, and there are differences across retroreflectors ( $\mathrm{E}$ and $\mathrm{M}$ performed best).

\subsection{Distance - Vibration}

To investigate our system's sensitivity to vibration, we conducted another test with the same procedure as the motion test, except that we generated a continuous swept-frequency vibration with the speaker diaphragm ( 2 to $2 \mathrm{kHz}$ over 20 seconds). Figure 13 shows the result, which indicates that our system is capable of detecting vibrations from retroreflectors at all distances up to $512 \mathrm{~m}$. The sensitivity characteristics are very similar to the motion test, with distance being a significant attenuation factor on the received signal power, with smaller variability across retroreflectors. Retroreflectors $\mathrm{E}$ and $\mathrm{M}$ again performed best.

\subsection{Main Findings}

To summarize, our main finding is that our system works in outdoor daylight conditions at ranges of up to $512 \mathrm{~m}$ for all retroreflectors we tested. This means one Vibrosight ++ setup could monitor a circular area $1 \mathrm{~km}$ in diameter. Our data also suggests that some retroreflectors could be sensed many kilometers away, assuming line of sight. However, range will decrease significantly with angles of incidence less than $45^{\circ}$, and if retroreflectors colors are mismatched with the laser (though red, white, and orange all appear to work well).

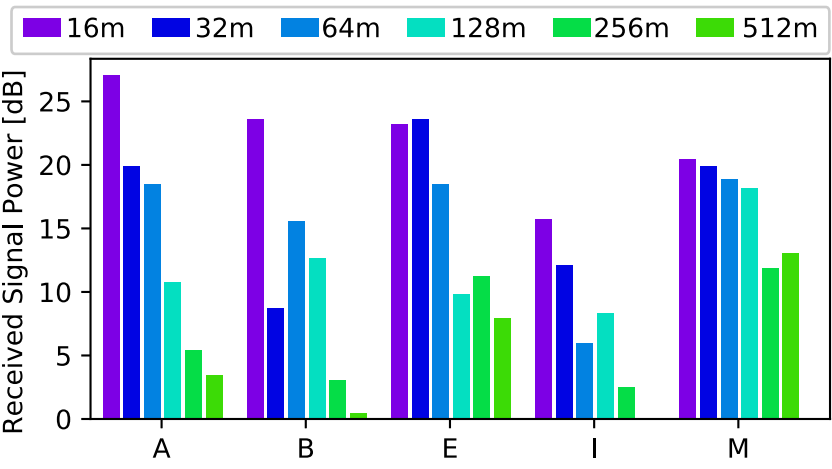

Figure 13: Received signal power from our distancevibration experiment using five exemplary retroreflectors. Refer to Figure 8 for letter mappings.

\section{EXAMPLE USES}

The practical applications for vibrometry in urban settings are immense. Given our signal is very accelerometer-like, we can generally enable all prior applications described in innumerable papers that have affixed powered accelerometers to objects and infrastructure. To illustrate this breadth of uses, we describe eleven example applications spread across three signal categories. These example applications are fully functional, powered by the detection pipelines in Section 5.5 and 5.6. Please also see our Video Figure.

\subsection{Vibration Sensing Examples}

The richest of our three signal categories is vibration. This signal allows for fine-grained detection, and more importantly, rejection of false-positive events that do not match the characteristic spectral signature of what we are trying to sense. We now describe four illustrative example uses:

7.1.1 Mailbox Utilization. Using the retroreflective USPS logo on the mailbox seen in Figure 7, it is very apparent when someone opens the hinged door to deposit a letter. A simple incrementing count could be used to track the number of visitors, which could inform mailbox placement, but perhaps more interestingly, be used to dynamically schedule pickup based on the estimated volume of mail pieces. We note in this example, the mailbox is immediately
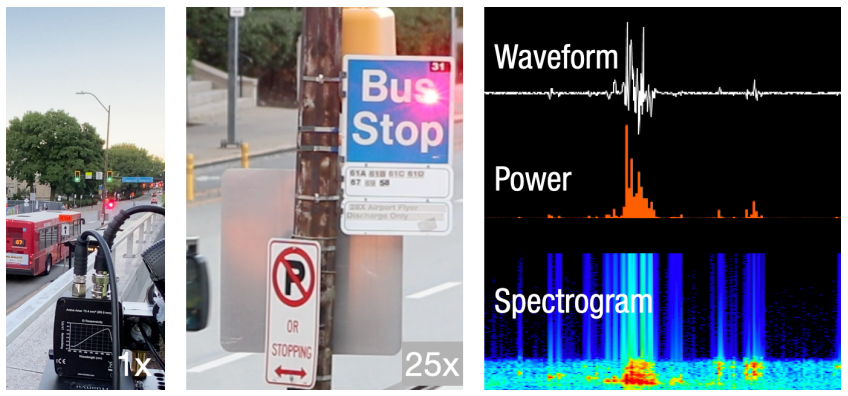

Figure 14: We threshold the power of unique frequency bands induced by a passing bus for detection. 

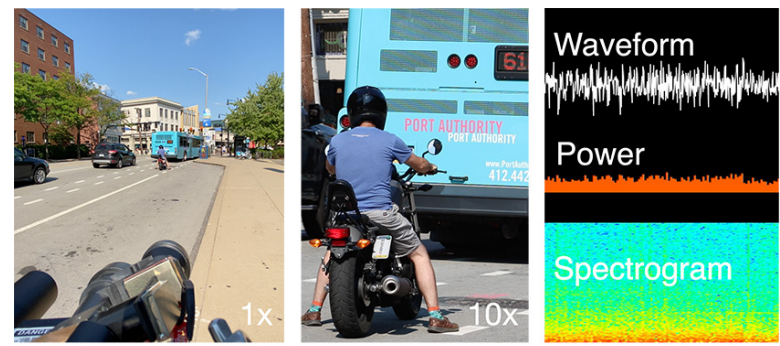

Motorcycle

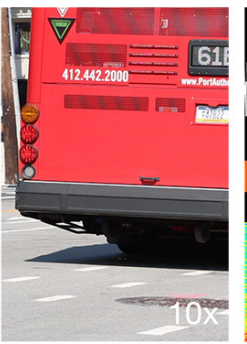

Bus

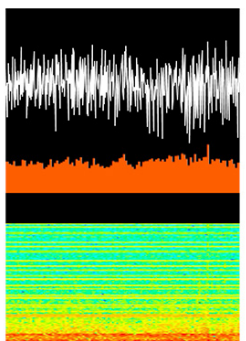

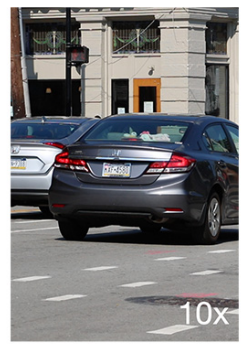

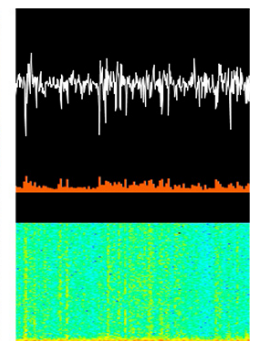

Sedan

Figure 15: Type of vehicle can also be detected based on their distinctive frequency components.

adjacent to a road; cars passing by also induce a vibration, but this spectral signature is very different and thus easily ignored.

7.1.2 Bus Arrival Detection and On-Time Performance Tracking. Bus stops are often demarcated by a sign, which serves both riders and drivers alike. We found that buses pulling up to a stop create a characteristic air front that causes the sign to flex and oscillate (Figure 14), much like a tuning fork. Smaller vehicles do not induce the same effect. Larger vehicles, such as trucks, can produce the same signal if they similarly decelerate to a stop next to the sign, though this event is less typical. However, at least in some cases, this signal could be useful to power live bus monitoring apps and perhaps track on-time performance.

7.1.3 Traffic Flow. Roads are painted with a wide variety of markings, including lanes, turns, and pedestrian crossings. Retroreflective glass beads are often incorporated into road paint so as to make the markings more visible at night. This means there are many millions of miles of "sensors" already installed on roads for us to use. As one example, we used a white shoulder line for detecting passing vehicles. The mass of a passing vehicle induces a low-frequency oscillation of the road surface that can be easily detected and then counted over time to measure traffic volume (Figure 16). Anecdotally, we also observed the shape of the waveform changes by vehicle velocity and type (number of axles, vehicle weight).

7.1.4 Vehicle Type Detection. Although we observed that vehicle type might be inferred directly from road markings (previous example), a more direct measurement is enabled by sensing engine
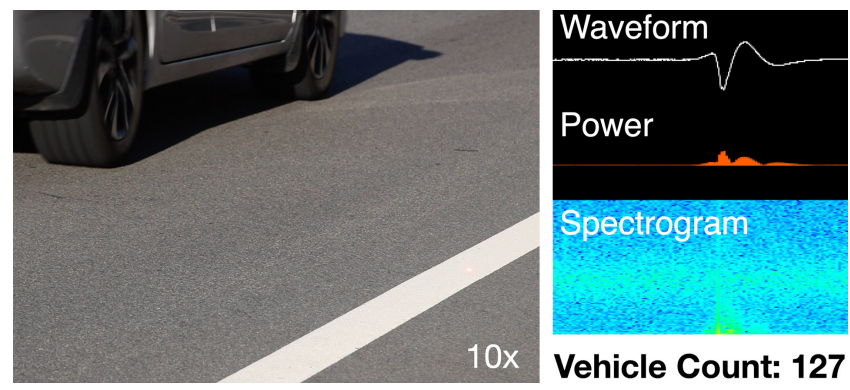

Vehicle Count: 127

Figure 16: We detect peaks on the raw waveform to count passing vehicles. vibration using the license plates of vehicles, which are retroreflective. As can be seen in Figure 15, the signals can be quite distinctive, and we were able to train a vehicle classifier (i.e., SVM with linear kernel) for cars, buses, and motorcycles. It may even be possible to infer engine type (inline 4 vs. V6, diesel vs. gasoline, etc.) and RPM, though we leave this to future work. A downside of sensing vehicles in this manner is that it requires rapid scanning of an intersection to find license plates and take a measurement, and thus it is likely that only subsampling of vehicle type is possible.

\subsection{Motion Sensing Examples}

We categorize low-frequency oscillations as movement, and built several example application classifiers not using spectral data, but rather mean power over time.

7.2.1 Block-scale Wind Monitoring. The large surface area of signs means they tend to catch the wind, causing deflection and oscillations, often at centimeter scales. Such gross changes can be easily captured by our sensor at very long ranges. As one example, we recorded a pole-mounted street sign affixed to a wooden utility pole on a breezy day. Figure 17 offers three example signals at different wind intensities. We found that wind signals often appeared as paired bumps - this is due to the wind deflecting the sign one way, and then having the sign return back to its neutral position.

7.2.2 Urban Tree Health. Like signage, trees also sway in response to wind. Interestingly, the sway frequency is correlated with leaf cover (i.e., biomass suspended on a trunk, forming a spring-mass system) and hydration (as water uptake affects the mass of the foliage) [12] (Figure 18). This signal could be used to detect anomalous

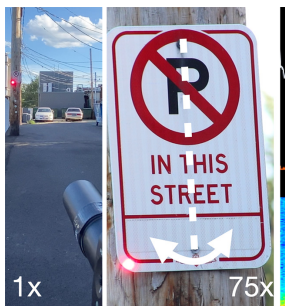

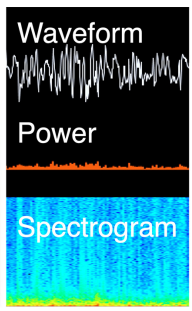

Still

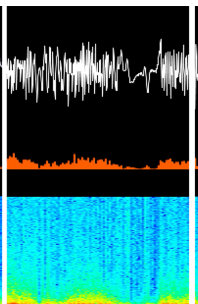

Breeze

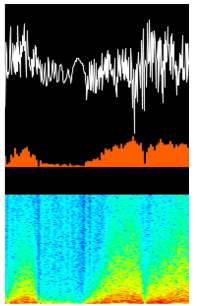

Gust
Figure 17: We can monitor wind conditions by tracking reflected signal power. 

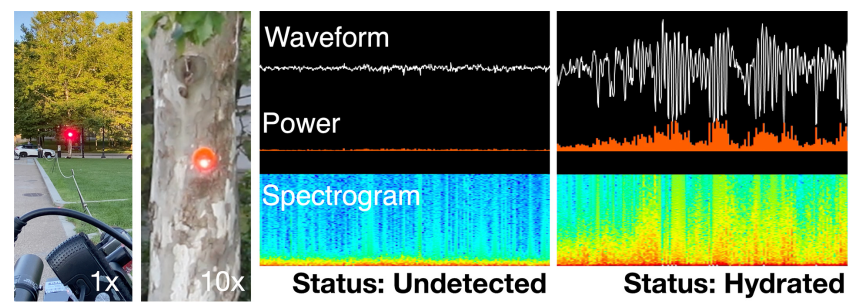

Figure 18: The water stress level of trees can be inferred by monitoring their sway frequency [12].

tree states in the cityscape to proactively dispatch care or schedule replacement (healthy urban trees reduce air pollution, provide shade, and beautify spaces, among many benefits). Although we did not build a fully working version of this example application (lacking ground-truth hydration data), we know that our system can readily detect the sway of trees using retroreflectors found on trunks (placed to help prevent collisions), and thus a model could certainly be built (as has been shown with physical accelerometer deployments [12].

7.2.3 Structural Health. IMUs have long been used to measure the movement and vibrations of infrastructure, such as buildings and bridges, to monitor structural health $[4,25]$. A change in signal often indicates a change in physical condition, triggering an onsite inspection. With our system, instead of having to deploy expensive physical sensors, we can simply use retroreflectors already attached to such structures as sensors. As one example, we recorded data from an overpass, and recorded the signals of passing cars (Figure 19). As with our tree health example, we did not have ground-truth data for this bridge that permitted a structural health inference, but we believe our recorded signals demonstrate this could be possible.
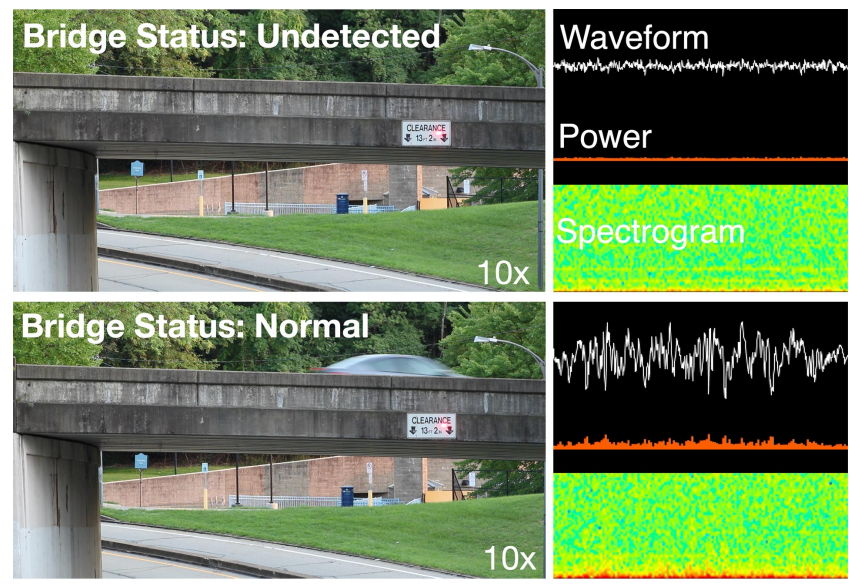

Figure 19: We can monitor bridge structural health using the magnitude and frequency of vibrations as vehicles cross.
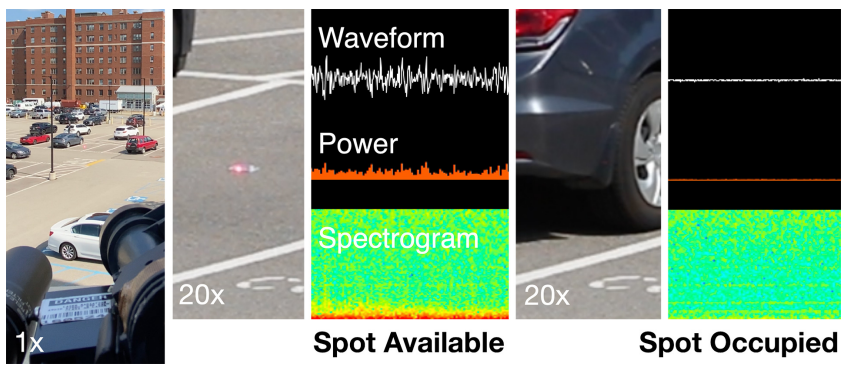

Figure 20: We detect parking spot occupancy based on occlusion of ground retroreflectors.

\subsection{Occlusion/Presence Sensing Examples}

Our third and final signal category is occlusion and presence sensing. In short, when our laser beam is blocked or the retroreflector is moved, our system sees a dramatic drop in received light intensity. This binary signal has no spectral or intensity information, though in some cases, the rate at which the signal turns on and off can be used as a useful signal.

7.3.1 Parking Spot Occupancy. Parking spots in cities are a limited resource, and many expensive sensing solutions are sold today to mitigate this issue. However, the high costs involved (often requiring installing a limited-life powered sensor in the pavement) has meant that publicly-owned, smart parking lots are comparatively rare today. With our system, passive road studs placed in the center of parking spots could be used as occlusion detectors (Figure 20). Given the sensing range of our unit, a whole parking lot could be sensed from a good vantage point.

7.3.2 Hyper-Local Weather Monitoring. Technologies like doppler weather radar are excellent at estimating rainfall volume down to areas around $1 \mathrm{~km}^{2}$ in size. For more localized measurements, physical weather stations with rainfall collectors must be deployed. With our system, we found that rainfall volume could be estimated using the brief signal interruptions caused by droplets passing through the laser path (Figure 21). As the distance to each retroreflector is
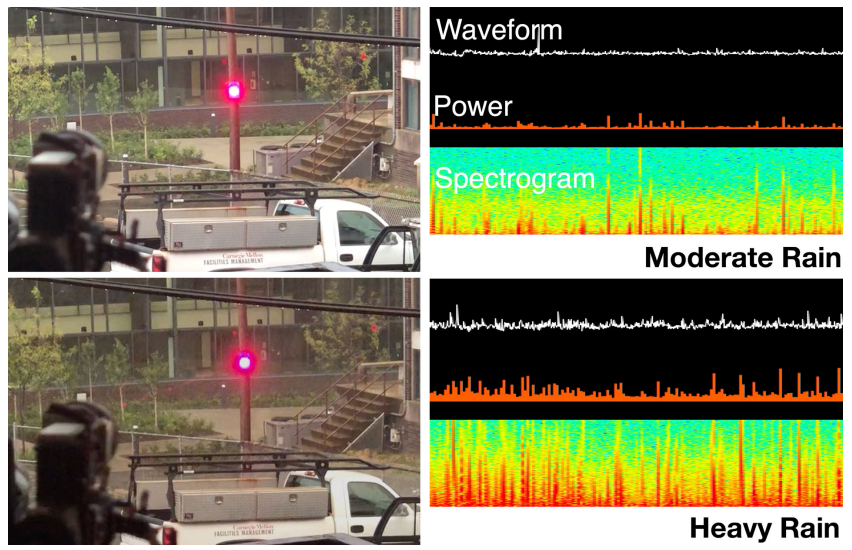

Figure 21: Rain intensity can be detected through tracking the power of non-DC frequency components. 


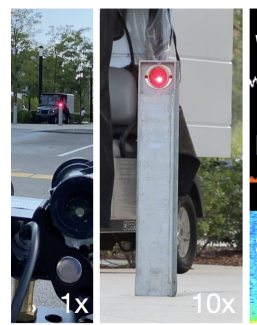

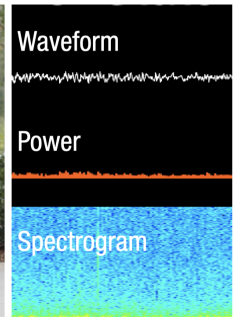

Barrier Up

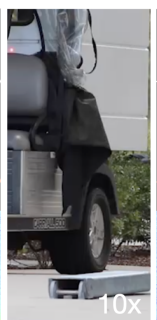

Barrier Down
Figure 22: We detect the road barrier's state with a simple threshold on the raw reflectance measurement.

known (see Depth Map in Section 5.4), the density of droplets can be estimated. This allows a single laser vibrometry base station to use its collection of found retroreflectors to sample the local rainfall in the immediate proximity. While we did not collect any formal data, we also saw that fog and air pollution had an attenuating effect on signal strength, which could power other weather-oriented use cases.

7.3.3 Sidewalk / Road Access. Powered or manually-operated movable traffic bollards are used to regulate access to pedestrian and vehicular routes. As these are traffic control devices, they typically feature a retroreflective marker, which we can use to detect state (Figure 22). With this data, we can potentially infer traffic flow to better manage city resources (e.g., street lighting).

7.3.4 Sanitation Collection. Finally, there are many objects that occupy the streetscape that are moveable, such as refuse dumpsters (Figure 23). By knowing their rough parked location, our system can scan to find them, and then periodically check to see if they have been moved or taken away. Such data could be used to push alerts to building management (e.g., "refuse collected") and perhaps better optimize collection schedules.

\subsection{System Accuracy}

All example applications in this section are fully functional, powered by the signal processing and detection pipeline discussed earlier in the paper. Please also refer to the Video Figure to see these example applications in action and the cleanliness of the live signals. With the data we collected during the development of these applications, we ran a basic classification study which demonstrated an average accuracy of functionally $100 \%$ - all errors were instability during state transitions, which could be trivially filtered with fine-tuned majority voting buffers. Rather than reporting this same accuracy number for the above sections, we believe our Figure and Video materials are more convincing. That said, there is still need for a longer-term deployment study, which will undoubtedly yield important insights about performance degradation over time, across a greater variety of environmental conditions, and robustness of the classifiers in practice.

\section{LIMITATIONS \& FUTURE WORK}

There are several important limitations of our approach that should be noted. The most obvious one is that our system relies on lineof-sight, which means if retroreflective signs and markers are not

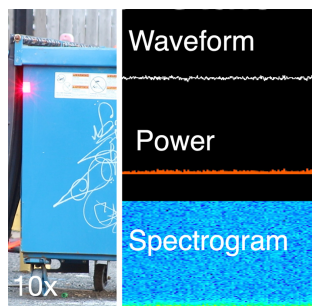

Dumpster Present

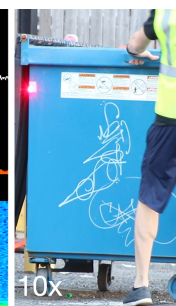

Moving

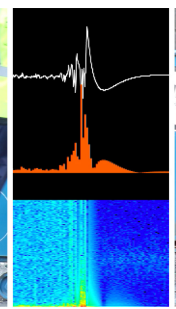

Removed

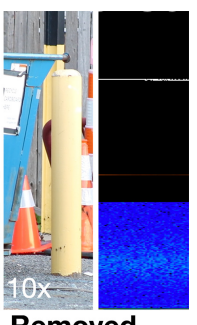

Figure 23: We detect when a dumpster is moved for collection with a simple threshold on the raw reflectance measurement.

visible to our sensor, we will not be able to capture signal. Fortunately, given the incredible density of retroreflective signs and markers distributed throughout cities, and the presence of street poles, advantageous viewpoints should not be hard to identify.

Second, the time-multiplexed nature of our current sensing mechanism means that our system is ill-suited to sense transient events (i.e., ones brief enough they can be missed while Vibrosight ++ is sampling other retroreflectors). Instead, our system is much better suited for continuous events (e.g., a tree swaying in the wind) or minute-scale changes to the environment (e.g., car parked in a spot, dumpster being emptied). We also note that our current implementation is not fast enough to rapidly scan and locate mobile objects (e.g., cars stopped at an intersection). While it may be possible to improve our retroreflector search speed with a faster pan-tilt platform, we believe there is more promise in building a system with multiple laser beams, either using several diodes or a diffraction rating, to overcome this limitation.

Third, even the longest sensing range that our system achieves is small compared to the size of cities. To get whole-city coverage is still going to take a lot of Vibrosight++ units with significant capital and labor costs. That said, given the wide-area nature of our technique, the cost per sensed point could be very low - perhaps just a few dollars. Additionally, the signals our system produces are fairly low level and offer tremendous flexibility for future uses without having to replace the deployed physical sensors (a la "software defined sensing" or "general purpose sensors" [28]).

Last but not least, it is important to acknowledge that our system has important privacy implications. The more that cities know about their populations, the more the data can be used for both good and ill effect. This is true of any powerful technology paradigm and wide-area sensing is no different. Although the captured accelerometry data itself is not particularly de-anonymizing, the location of where that data came from could be (e.g., someone's vehicle license plate parked in their driveway). For this reason, extreme care must be taken with recorded data, in a similar manner to security cameras today. Edge machine learning, where raw data is never transmitted to the cloud and instead processed locally and aggregated, is an obvious layer of protection that we endorse and use in many of our other research projects. We hope to release a follow-up paper that more deeply discusses the privacy properties of Vibrosight++ to stimulate discussion in this area, which we believe could be valuable for both city planners and HCI practitioners. 


\section{CONCLUSION}

We have described a new, long-range, laser sensing system called Vibrosight ++ that co-opts retroreflective signs and markers as sensors for smart city applications. This sensing approach dramatically reduces deployment and maintenance costs, and a the same time, generalizes across a wide array of sensing applications that can improve cities. Our approach is mainly based on laser vibrometry with which we can detect minute motion and vibration on retroreflectors, which often carry rich signals that can be used for traffic sensing, vehicle type detection, infrastructure health monitoring, and beyond. Through a series of studies, we have characterized our system's performance across many factors, the results of which indicate that our system provides a feasible path to practical city-scale activity sensing for future smart cities.

\section{REFERENCES}

[1] American National Standards Institute. 2014. ANSI Z136.1 - Safe Use of Lasers. https://www.lia.org/resources/laser-safety-information/laser-safetystandards/ansi-z136-standards/z136-1 Retrieved January 10, 2021

[2] Array of Things. 2020. Project Information. https://arrayofthings.github.io Retrieved January 10, 2021

[3] Kridsakron Auynirundronkool, Nengcheng Chen, Caihua Peng, Chao Yang, Jianya Gong, and Chaowalit Silapathong. 2012. Flood detection and mapping of the Thailand Central plain using RADARSAT and MODIS under a sensor web en vironment. International fournal of Applied Earth Observation and Geoinformation 14, 1 (2012), 245 - 255. https://doi.org/10.1016/j.jag.2011.09.017

[4] Yuequan Bao, James L Beck, and Hui Li. 2011. Compressive sampling for accelerometer signals in structural health monitoring. Structural Health Monitoring 10, 3 (2011), 235-246. https://doi.org/10.1177/1475921710373287

[5] Johan Barthélemy, Nicolas Verstaevel, Hugh Forehead, and Pascal Perez. 2019 Edge-Computing Video Analytics for Real-Time Traffic Monitoring in a Smart City. Sensors 19, 9 (May 2019), 2048. https://doi.org/10.3390/s19092048

[6] Juan P Bello, Claudio Silva, Oded Nov, R Luke DuBois, Anish Arora, Justin Salamon, Charles Mydlarz, and Harish Doraiswamy. 2019. Sonyc: A system for monitoring, analyzing, and mitigating urban noise pollution. Commun. ACM 62 , 2 (2019), 68-77.

[7] Austin Lyons Bischoff and Darcy M Bullock. 2002. Sign retroreflectivity study. Foint Transportation Research Program (01 2002). https://doi.org/10. $5703 / 1288284313313$

[8] Jillian Carr and Jennifer L Doleac. 2016. The geography, incidence, and underreporting of gun violence: new evidence using ShotSpotter data. Incidence, and Underreporting of Gun Violence: New Evidence Using Shotspotter (2016) https://doi.org/10.2139/ssrn.2770506

[9] Paolo Castellinim, Milena Martarelli, and Enrico Primo Tomasini. 2006. Laser Doppler Vibrometry: Development of advanced solutions answering to technology's needs. Mechanical Systems and Signal Processing 20, 6 (2006), 1265 1285. https://doi.org/10.1016/j.ymssp.2005.11.015 Special Issue: Laser Doppler Vibrometry.

[10] Yiheng Chen and Dawei Han. 2018. Water quality monitoring in smart city: A pilot project. Automation in Construction 89 (2018), 307 - 316. https://doi.org/10 1016/j.autcon.2018.02.008

[11] Emilio Chuvieco. 2016. Fundamentals of satellite remote sensing: An environmental approach. CRC press.

[12] Dominick M. Ciruzzi and Steven P. Loheide II. 2019. Monitoring Tree Sway as an Indicator of Water Stress. Geophysical Research Letters 46, 21 (2019), 12021-12029. https://doi.org/10.1029/2019GL084122

[13] Code of Federal Regulations. 2011. Title 49 §393.11 - Lamps and reflective devices. https://www.govinfo.gov/app/details/CFR-2011-title49-vol5/CFR-2011title49-vol5-sec393-11 Retrieved January 10, 2021.

[14] Cristina Cristalli, Nicola Paone, and R. M. Rodríguez. 2006. Mechanical fault detection of electric motors by laser vibrometer and accelerometer measurements. Mechanical Systems and Signal Processing 20, 6 (2006), 1350 - 1361. https://doi. org/10.1016/j.ymssp.2005.11.013 Special Issue: Laser Doppler Vibrometry.

[15] Amr S El-Wakeel, Jin Li, Muhammed T Rahman, Aboelmagd Noureldin, and Hossam S Hassanein. 2017. Monitoring road surface anomalies towards dynamic road mapping for future smart cities. In IEEE Global Conference on Signal and Information Processing (GlobalSIP '17). IEEE, 828-832. https://doi.org/10.1109/ GlobalSIP.2017.8309076

[16] William Emery and Adriano Camps. 2017. Introduction to satellite remote sensing:

atmosphere, ocean, land and cryosphere applications. Elsevier.
[17] Jennifer Gabrys. 2014. Programming Environments: Environmentality and Citizen Sensing in the Smart City. Environment and Planning D: Society and Space 32,
1 (2014), 30-48. https://doi.org/10.1068/d16812

[18] Guido Giuliani, Simone Bozzi-Pietra, and Silvano Donati. 2002. Self-mixing laser diode vibrometer. Measurement Science and Technology 14, 1 (nov 2002), 24-32. https://doi.org/10.1088/0957-0233/14/1/304

[19] Earl E. Gossard. 1988. Measuring Drop-Size Distributions in Clouds with a Clear-Air-Sensing Doppler Radar. Journal of Atmospheric and Oceanic Technology 5, 5 (oct 1988), 640 - 649. https://doi.org/10.1175/1520-0426(1988)005<0640: MDSDIC>2.0.CO;2

[20] Daniel Granlund and Robert Brännström. 2012. Smart City: The smart sewerage. In 37th Annual IEEE Conference on Local Computer Networks - Workshops (LCNW '12). IEEE, 856-859. https://doi.org/10.1109/LCNW.2012.6424074

[21] Hadi Habibzadeh, Zhou Qin, Tolga Soyata, and Burak Kantarci. 2017. LargeScale Distributed Dedicated- and Non-Dedicated Smart City Sensing Systems. IEEE Sensors fournal 17, 23 (2017), 7649-7658. https://doi.org/10.1109/JSEN.2017. 2725638

[22] Gerhard Hancke, Bruno Silva, and Gerhard Hancke, Jr. 2012. The Role of Advanced Sensing in Smart Cities. Sensors 13, 1 (Dec 2012), 393-425. https: //doi.org/10.3390/s130100393

[23] Roy Henderson and Karl Schulmeister. 2003. Laser safety. CRC Press.

[24] Zhiwen Hu, Zixuan Bai, Yuzhe Yang, Zijie Zheng, Kaigui Bian, and Lingyang Song. 2019. UAV Aided Aerial-Ground IoT for Air Quality Sensing in Smart City: Architecture, Technologies, and Implementation. IEEE Network 33, 2 (2019), 14-22. https://doi.org/10.1109/MNET.2019.1800214

[25] Sukun Kim, Shamim Pakzad, David Culler, James Demmel, Gregory Fenves, Steve Glaser, and Martin Turon. 2006. Wireless Sensor Networks for Structural Health Monitoring. In Proceedings of the 4th International Conference on Embedded Networked Sensor Systems (Boulder, Colorado, USA) (SenSys '06). Association for Computing Machinery, New York, NY, USA, 427-428. https://doi.org/10.1145/ 1182807.1182889

[26] Gierad Laput, Walter S. Lasecki, Jason Wiese, Robert Xiao, Jeffrey P. Bigham, and Chris Harrison. 2015. Zensors: Adaptive, Rapidly Deployable, Human-Intelligent Sensor Feeds. In Proceedings of the 33rd Annual ACM Conference on Human Factors in Computing Systems (Seoul, Republic of Korea) (CHI '15). Association for Computing Machinery, New York, NY, USA, 1935-1944. https://doi.org/10. 1145/2702123.2702416

[27] Gierad Laput, Robert Xiao, and Chris Harrison. 2016. ViBand: High-Fidelity BioAcoustic Sensing Using Commodity Smartwatch Accelerometers. In Proceedings of the 29th Annual Symposium on User Interface Software and Technology (Tokyo, Japan) (UIST '16). Association for Computing Machinery, New York, NY, USA, 321-333. https://doi.org/10.1145/2984511.2984582

[28] Gierad Laput, Yang Zhang, and Chris Harrison. 2017. Synthetic Sensors: Towards General-Purpose Sensing. In Proceedings of the 2017 CHI Conference on Human Factors in Computing Systems (Denver, Colorado, USA) (CHI '17). Association for Computing Machinery, New York, NY, USA, 3986-3999. https://doi.org/10.1145/ 3025453.3025773

[29] Fabio Leccese, Marco Cagnetti, and Daniele Trinca. 2014. A Smart City Application: A Fully Controlled Street Lighting Isle Based on Raspberry-Pi Card, a ZigBee Sensor Network and WiMAX. Sensors 14, 12 (Dec 2014), 24408-24424. https://doi.org/10.3390/s141224408

[30] W. H. Leong, Wiesław Jerzy Staszewski, B. C. Lee, and Fabrizio Scarpa. 2005. Structural health monitoring using scanning laser vibrometry: III. Lamb waves for fatigue crack detection. Smart Materials and Structures 14, 6 (oct 2005), 1387-1395. https://doi.org/10.1088/0964-1726/14/6/031

[31] Zhong-Xian Li, Xiao-Ming Yang, and Zongjin Li. 2006. Application of CementBased Piezoelectric Sensors for Monitoring Traffic Flows. Fournal of Transportation Engineering 132, 7 (2006), 565-573. https://doi.org/10.1061/(ASCE)0733947X(2006)132:7(565)

[32] Shunlin Liang and Jindi Wang. 2019. Advanced remote sensing: terrestrial information extraction and applications. Academic Press.

[33] Shi-Wei Lo, Jyh-Horng Wu, Fang-Pang Lin, and Ching-Han Hsu. 2015. Visual Sensing for Urban Flood Monitoring. Sensors 15, 8 (Aug 2015), 20006-20029. https://doi.org/10.3390/s150820006

[34] Jon Derek Loftis, David Forrest, Sridhar Katragadda, Kyle Spencer, Tammie Organski, Cuong Nguyen, and Sokwoo Rhee. 2018. StormSense: A New Integrated Network of IoT Water Level Sensors in the Smart Cities of Hampton Roads, VA. Marine Technology Society fournal 52, 2 (2018), 56-67. https://doi.org/10.4031/ MTSJ.52.2.7

[35] Xianghong Ma, Lawrence Bergman, and Alexander Vakakis. 2001. Identification of Bolted Joints Through Laser Vibrometry. Journal of Sound and Vibration 246, 3 (2001), 441 - 460. https://doi.org/10.1006/jsvi.2001.3573

[36] Mousumi Majumder, Tarun Kumar Gangopadhyay, Ashim Kumar Chakraborty, Kamal Dasgupta, and Dipak Kumar Bhattacharya. 2008. Fibre Bragg gratings in structural health monitoring-Present status and applications. Sensors and Actuators A: Physical 147, 1 (2008), 150 - 164. https://doi.org/10.1016/j.sna.2008. 04.008

[37] MetroCount Inc. 2020. Metrocount. https://metrocount.com Retrieved January 10, 2021. 
[38] Saraju P Mohanty, Uma Choppali, and Elias Kougianos. 2016. Everything you wanted to know about smart cities: The Internet of things is the backbone. IEEE Consumer Electronics Magazine 5, 3 (2016), 60-70. https://doi.org/10.1109/MCE. 2016.2556879

[39] Wongi Na and Jongdae Baek. 2018. A Review of the Piezoelectric Electromechanical Impedance Based Structural Health Monitoring Technique for Engineering Structures. Sensors 18, 5 (Apr 2018), 1307. https://doi.org/10.3390/s18051307

[40] Christophe Ishimwe Ngabo and Omar El Beqqali. 2018. 3D tilt sensing by using accelerometer-based wireless sensor networks: Real case study: Application in the smart cities. In International Conference on Intelligent Systems and Computer Vision (ISACV'18). IEEE, 1-8. https://doi.org/10.1109/ISACV.2018.8354013

[41] Charith Perera, Arkady Zaslavsky, Peter Christen, and Dimitrios Georgakopoulos. 2014. Sensing as a service model for smart cities supported by Internet of Things. Transactions on Emerging Telecommunications Technologies 25, 1 (2014), 81-93. https://doi.org/10.1002/ett.2704

[42] Polytec Inc. 2020. Product Information. https://www.polytec.com/us/vibrometry/ products/single-point-vibrometers/vibrogo/ Retrieved January 10, 2021

[43] Mark Potosnak, Pinaki Banerjee, Rajesh Sankaran, Veerabhadra Kotamarthi, Robert Jacob, Peter Beckman, and Charlie Catlett. 2018. Array of Things: Characterizing low-cost air quality sensors for a city-wide instrument. Earth and Space Science Open Archive (2018), 1. https://doi.org/10.1002/essoar.10500209.1

[44] Manaswi Saha, Michael Saugstad, Hanuma Teja Maddali, Aileen Zeng, Ryan Holland, Steven Bower, Aditya Dash, Sage Chen, Anthony Li, Kotaro Hara, and Jon Froehlich. 2019. Project Sidewalk: A Web-Based Crowdsourcing Tool for Collecting Sidewalk Accessibility Data At Scale. In Proceedings of the 2019 CHI Conference on Human Factors in Computing Systems (Glasgow, Scotland UK) (CHI '19). Association for Computing Machinery, New York, NY, USA, 1-14 https://doi.org/10.1145/3290605.3300292

[45] Nobuhiro Shimoi, C. Cuadra, Madokoroa Hirokazu, and Masahiro Saijo. 2012 Simple smart piezoelectric bolt sensor for structural monitoring of bridges. Int. 7 . Instrumentation Science 1, 5 (2012), 78-83. https://doi.org/10.5923/j.instrument 20120105.03

[46] Robert L Showen, Robert B Calhoun, and Jason W Dunham. 2009. Acoustic location of gunshots using combined angle of arrival and time of arrival measurements. US Patent 7,474,589.

[47] Wei Sun, Tuochao Chen, Jiayi Zheng, Zhenyu Lei, Lucy Wang, Benjamin Steeper, Peng He, Matthew Dressa, Feng Tian, and Cheng Zhang. 2020. VibroSense: Recognizing Home Activities by Deep Learning Subtle Vibrations on an Interior Surface of a House from a Single Point Using Laser Doppler Vibrometry. Proc ACM Interact. Mob. Wearable Ubiquitous Technol. 4, 3, Article 96 (Sept. 2020), 28 pages. https://doi.org/10.1145/3411828
[48] Saiganesh Swaminathan, Jonathon Fagert, Michael Rivera, Andrew Cao, Gierad Laput, Hae Young Noh, and Scott E. Hudson. 2020. OptiStructures: Fabrication of Room-Scale Interactive Structures with Embedded Fiber Bragg Grating Optical Sensors and Displays. Proc. ACM Interact. Mob. Wearable Ubiquitous Technol. 4, 2 , Article 50 (June 2020), 21 pages. https://doi.org/10.1145/3397310

[49] Francisco Sánchez-Rosario, David Sánchez-Rodríguez, Jesús B Alonso-Hernández, Carlos M Travieso-González, Itziar Alonso-González, Carlos Ley-Bosch, Carlos Ramírez-Casañas, and Miguel A Quintana-Suárez. 2015. A low consumption real time environmental monitoring system for smart cities based on ZigBee wireless sensor network. In International Wireless Communications and Mobile Computing Conference (IWCMC '15). IEEE, 702-707. https://doi.org/10.1109/IWCMC.2015. 7289169

[50] The Free Encyclopedia Wikipedia. 2020. Street furniture. https://en.wikipedia. org/w/index.php?title=Street_furniture\&oldid=985712859 Retrieved January 10, 2021.

[51] Deepak Vasisht, Zerina Kapetanovic, Jongho Won, Xinxin Jin, Ranveer Chandra, Sudipta Sinha, Ashish Kapoor, Madhusudhan Sudarshan, and Sean Stratman. 2017. FarmBeats: An IoT Platform for Data-Driven Agriculture. In 14th USENIX Symposium on Networked Systems Design and Implementation (NSDI 17). USENIX Association, Boston, MA, 515-529. https://www.usenix.org/conference/nsdi17/ technical-sessions/presentation/vasisht

[52] Meisong Wang, Charith Perera, Prem Prakash Jayaraman, Miranda Zhang, Peter Strazdins, R. K. Shyamsundar, and Rajiv Ranjan. 2016. City Data Fusion: Sensor Data Fusion in the Internet of Things. International fournal of Distributed Systems and Technologies (IFDST) 7, 1 (2016), 15-36. https://doi.org/10.4018/IJDST. 2016010102

[53] World Sensing. 2020. Traffic Flow Monitoring. https://www.worldsensing.com/ Retrieved January 10, 2021.

[54] Andrea Zanella, Nicola Bui, Angelo Castellani, Lorenzo Vangelista, and Michele Zorzi. 2014. Internet of Things for Smart Cities. IEEE Internet of Things fournal 1, 1 (2014), 22-32. https://doi.org/10.1109/JIOT.2014.2306328

[55] Zensors Inc. 2020. Zensors. https://www.zensors.com Retrieved January 10, 2021.

[56] Guifu Zhang, Richard J Doviak, Dusan S Zrnic, Jerry Crain, David Staiman, and Yasser Al-Rashid. 2009. Phased Array Radar Polarimetry for Weather Sensing: A Theoretical Formulation for Bias Corrections. IEEE Transactions on Geoscience and Remote Sensing 47, 11 (2009), 3679-3689. https://doi.org/10.1109/TGRS.2009. 2029332

[57] Yang Zhang, Gierad Laput, and Chris Harrison. 2018. Vibrosight: Long-Range Vibrometry for Smart Environment Sensing. In Proceedings of the 31st Annual ACM Symposium on User Interface Software and Technology (Berlin, Germany) (UIST '18). Association for Computing Machinery, New York, NY, USA, 225-236. https://doi.org/10.1145/3242587.3242608 